\title{
Correlation of plasma concentration and adverse effects of bosutinib: standard dose or dose-escalation regimens of bosutinib treatment for patients with chronic myeloid leukemia
}

Akiko Mita ${ }^{1 \dagger}$, Maiko Abumiya ${ }^{2 \dagger}$, Masatomo Miura ${ }^{2}$, Takenori Niioka², Saori Takahashi ${ }^{3}$, Tomoko Yoshioka ${ }^{1}$, Yoshihiro Kameoka ${ }^{1,3}$ and Naoto Takahashi ${ }^{1 *}$ (])

\begin{abstract}
Purpose: To investigate the exposure-toxicity relationship of bosutinib and to identify the target trough plasma concentration $\left(C_{0}\right)$.

Methods: The toxicity and $\mathrm{C}_{0}$ of bosutinib in Japanese chronic myeloid leukemia (CML) patients were monitored every 2 weeks for the first 3 months of treatment, and subsequently once a month during the 6 months after beginning $500 \mathrm{mg} /$ day of standard dose (SD group, $\mathrm{n}=10$ ) or beginning $100 \mathrm{mg} /$ day and increased by $100 \mathrm{mg}$ every 2 weeks of dose escalation (DE group, $n=15$ ).

Results: Nine of 10 patients (90\%) in the SD group were not able to continue bosutinib therapy without interruption due to adverse events, compared to 2 patients (13.5\%) in the DE group. The total duration of treatment interruption was 35 and 14 days in the SD and DE groups, respectively. The median time until liver dysfunction or diarrhea was day 28 and day 1 in the SD group, and day 53.5 and day 19 in the DE group, respectively. The cumulative dose of bosutinib was comparable between the SD and DE groups (51,700 vs. 53,550 mg, respectively). At 6 months, the median $C_{0}$ was $63.7 \mathrm{ng} / \mathrm{mL}$ and $63.0 \mathrm{ng} / \mathrm{mL}$ in the SD and DE groups, respectively. Liver dysfunction (all grades) and diarrhea (> grade 2) were prevalent in quartile 4 of $C_{0}(>91.0 \mathrm{ng} / \mathrm{mL})$, as calculated by the total $C_{0}$ distribution.
\end{abstract}

Conclusions: The DE regimen was better suited to avoid treatment interruption. The daily dose of bosutinib might be adjusted based on target $C_{0}$ to avoid adverse events by therapeutic drug monitoring in general practice.

Keywords: Bosutinib, Diarrhea, Liver dysfunction, Trough concentration, Chronic myeloid leukemia

\section{Background}

Bosutinib is a second-generation tyrosine kinase inhibitor (TKI) for the Src and Abelson (Abl) kinases, which was originally developed for the treatment of patients

\footnotetext{
*Correspondence: naotot@doc.med.akita-u.ac.jp

${ }^{\dagger}$ Akiko Mita and Maiko Abumiya contributed equally to this work

${ }^{1}$ Department of Hematology, Nephrology, and Rheumatology, Akita

University Graduate School of Medicine, 1-1-1 Hondo, Akita City, Akita 010-8543, Japan

Full list of author information is available at the end of the article
}

with chronic phase (CP), accelerated phase, and blast phase Philadelphia chromosome-positive chronic myeloid leukemia (CML), who developed resistance or intolerance to prior TKIs such as imatinib, nilotinib, and dasatinib, or for whom other TKIs are not regarded as appropriate treatment options due to comorbidities [1-3]. In addition, in the Bosutinib Efficacy and Safety in Newly Diagnosed CML (BELA) trial and the Bosutinib trial in First-line chrOnic myelogenous leukemia tREatment (BFORE) trial, the achievement rate of a major 
molecular response (MMR) was significantly higher, and the median times to achieve MMR was significantly shorter, in patients taking bosutinib than in those taking imatinib $[4,5]$. Recently, bosutinib was approved as a first-line therapy for CML patients by the US Food and Drug Administration (FDA).

In TKI therapy for CML patients, therapeutic drug monitoring (TDM) is a new strategy for optimizing dosage to achieve faster and deeper responses, such as an MMR [6]. Although this strategy could increase efficacy and/or decrease toxicity from TKI therapy, it is important to confirm the proposed target plasma concentrations, especially in medications with narrow therapeutic windows. Namely, the dosage of TKI could be adjusted based on target plasma concentration to maximize efficacy and to minimize the incidence of adverse events (AEs). For CML patients taking imatinib, the target trough plasma concentration $\left(\mathrm{C}_{0}\right)$ are reported to be set above $1000 \mathrm{ng} / \mathrm{mL}$ to maximize efficacy. Bosutinib exposure and $C_{0}$ calculated from pooled plasma samples within 1 month after initiation of bosutinib therapy have also been reported to be associated with an achievement rate of MMR within 12 months [7]; however, target bosutinib $\mathrm{C}_{0}$ is uncertain. In addition, patients with a higher bosutinib $\mathrm{C}_{0}$ tended to have an elevated risk of bosutinibinduced diarrhea [8], which is the most frequent adverse event due to bosutinib [1-5, 7-10]. These results show that TDM may be necessary for bosutinib. However, these pharmacokinetic results from phase I-III trials [7, 8] were limited in that they evaluated clinical responses, such as MMR at 12 months, as well as toxicities, by only examining bosutinib exposure within 1 month after administration. The dose reduction of bosutinib is typically a result of AEs, which was shown to occur in about $39 \%$ of patients assessed for at least 1 year during a phase III trial [4].

In the present study, we monitored $\mathrm{C}_{0}$ of bosutinib in Japanese CML patients during the 6 months after beginning the standard dose or dose escalation of bosutinib, and investigated the relationships between bosutinib exposure and toxicities, especially diarrhea and liver dysfunction.

\section{Methods}

\section{Patients and protocols}

The 25 Japanese CML patients enrolled in this study were either resistant to, or intolerant of, imatinib, except for 1 patient with newly diagnosed de novo CML. All patients were treated at the Akita University Hospital between July 2009 and August 2017. The study was conducted according to the principles of the Declaration of Helsinki. This study was approved by the Ethics Committee of Akita University Graduate School of Medicine (no. 1140).
Each participant provided informed consent and signed a human subject institutional review board consent form. The first 10 patients among the 25 patients were treated with the standard dose of bosutinib (500 mg, once daily) and the other 15 patients were treated using a dose escalation regimen. Patient characteristics at the initial bosutinib administration (baseline) are listed in Table 1. During the study period, patients were not administered proton pump inhibitors and/or drugs or foods known to affect CYP3A or P-glycoprotein function. Details of the standard dose (SD) regimen included the following. Initially, bosutinib was orally administered $500 \mathrm{mg}$ once daily at 08:00 after breakfast. After that, the maintenance dosage of bosutinib was adjusted in 100-mg increments, based on patient condition. Basically, dose reductions of bosutinib were conducted based on AE grade, for each side effect. For example, at the time of onset for grade 1-2 AEs, the bosutinib therapy was continued by using the same dose with an appropriate supporting therapy while observing each patient's condition. At the time of onset for grade $3 \mathrm{AEs}$, the bosutinib therapy was temporarily discontinued until each $\mathrm{AE}$ was asymptomatic. After that, the patient received the same dose, or the previous dose reduced by $100 \mathrm{mg}$.

Details of the dose escalation (DE) regimen included the following. Initially, bosutinib was orally administered at $100 \mathrm{mg}$ once daily at 08:00 after breakfast during the first 2 weeks of therapy initiation. On day 15 after beginning therapy, bosutinib was increased to $200 \mathrm{mg}$ once daily based on each patient's condition; subsequently, every 2 weeks, bosutinib was increased by $100 \mathrm{mg}$ to a maximum of $500 \mathrm{mg} /$ day based on patient condition. The final bosutinib dose is shown in Table 2. For AEs, toxicity grades were determined using the Common Terminology Criteria for Adverse Events (CTCAE) version 4.0.

During bosutinib administration, patients had blood collected at regular intervals for the monitoring of side effects. Namely, bosutinib $\mathrm{C}_{0}$ was monitored at just prior to oral administration or $25 \pm 1 \mathrm{~h}$ after administration for outpatients, in 2-week intervals for the first 3 months after beginning treatment, then once a month during the rest of the 6-month treatment period. A total of 136 samples of bosutinib plasma concentrations were collected in this study. Plasma was isolated by centrifugation at $1900 \times g$ for $15 \mathrm{~min}$ and was stored at $-40{ }^{\circ} \mathrm{C}$ until analyzed.

\section{Analysis of bosutinib plasma concentrations}

Plasma concentrations of bosutinib were measured by high-performance liquid chromatography (HPLC). Following the addition of erlotinib $(5 \mathrm{ng} / 10 \mu \mathrm{L}$ of methanol) as an internal standard to a $100 \mu \mathrm{L}$ plasma sample, the plasma sample was diluted with $900 \mu \mathrm{L}$ of water and 
Table 1 Clinical characteristics of patients administered bosutinib

\begin{tabular}{|c|c|c|c|}
\hline \multirow[t]{2}{*}{ Characteristics } & \multicolumn{2}{|l|}{ Frequency } & \multirow[t]{2}{*}{$P$ value } \\
\hline & Standard dose & Dose escalation & \\
\hline Total number & 10 & 15 & \\
\hline Female: male & $5: 5$ & $8: 7$ & \\
\hline Age (year) & $63.0(34.0-78.0)$ & $55.0(19.0-81.0)$ & 0.183 \\
\hline Phase, CP: AP: BP & $7: 2: 1$ & 15:0:0 & \\
\hline Reason of taking bosutinib, TKI-R: TKI-Int: de novo CML & 4:5:1 & 9:6:0 & \\
\hline Body weight (kg) & $59.9(40.8-69.1)$ & $53.0(44.1-86.3)$ & 0.889 \\
\hline \multicolumn{4}{|l|}{ Laboratory test values } \\
\hline White blood cell $\left({ }^{*} 10^{3} / \mathrm{mm}^{3}\right)$ & $8.5(3.6-190.7)$ & $6.1(1.3-13.8)$ & 0.332 \\
\hline Hemoglobin (g/dL) & $10.3(8.2-14.2)$ & $12.1(8.3-14.8)$ & 0.052 \\
\hline Platelet $\left({ }^{*} 10^{4} / \mathrm{mm}^{3}\right)$ & $29.5(12.1-326.0)$ & $19.4(12.8-162.0)$ & 0.120 \\
\hline Aspartate transaminase (IU/L) & $23.0(14.0-56.0)$ & $24.0(10.0-47.0)$ & 0.487 \\
\hline Alanine transaminase $(I \cup / L)$ & $17.5(9.0-65.0)$ & $23.0(7.0-34.0)$ & 0.232 \\
\hline Serum albumin (g/dL) & $4.1(3.7-4.3)$ & $4.2(3.0-5.2)$ & 0.179 \\
\hline Total bilirubin (mg/dL) & $0.6(0.2-0.9)$ & $0.6(0.3-2.7)$ & 0.557 \\
\hline Serum creatinine (mg/dL) & $0.8(0.5-0.9)$ & $0.7(0.4-1.7)$ & 0.803 \\
\hline Lipase (IU/L) & $21.5(5.0-75.0)$ & $18.0(6.0-38.0)$ & 0.781 \\
\hline
\end{tabular}

Data presented as number or median (minimum-maximum)

$T K I$ tyrosine kinase inhibitor, $R$ resistance, Int intolerance

Table 2 Patient disposition and dose intensity for the study treatment

\begin{tabular}{|c|c|c|c|c|}
\hline \multicolumn{2}{|l|}{ All patients during day 1 to day 180} & \multirow{2}{*}{$\begin{array}{c}\text { Standard dose }(\mathbf{n}=\mathbf{1 0}) \\
9(90.0 \%)\end{array}$} & \multirow{2}{*}{$\begin{array}{c}\text { Dose escalation }(\mathbf{n}=\mathbf{1 5}) \\
2(13.3 \%)\end{array}$} & \multirow{2}{*}{$\begin{array}{r}\boldsymbol{P} \text { value } \\
<0.001\end{array}$} \\
\hline Interruption, n (\%) & & & & \\
\hline Diarrhea & & $9(90.0 \%)$ & $0(0.0 \%)$ & \\
\hline Liver dysfunctions & & $5(50.0 \%)$ & $2(13.3 \%)$ & \\
\hline Skin rash & & $2(20.0 \%)$ & $0(0.0 \%)$ & \\
\hline Cytopenia & & $3(30.0 \%)$ & $0(0.0 \%)$ & \\
\hline Elevation of lipase & & $1(10.0 \%)$ & $0(0.0 \%)$ & \\
\hline \multicolumn{2}{|c|}{ Median (range) day to the first interruption of bosutinib } & $17.5(8-180)$ & $180(35-180)$ & $<0.001$ \\
\hline \multicolumn{2}{|c|}{ Total median (range) duration of treatment interruption (days) } & $35(8-81)$ & $14(14-14)$ & 0.013 \\
\hline \multicolumn{2}{|l|}{ Dose reduction, n (\%) } & $8(80.0 \%)$ & $8(53.3 \%)$ & 0.182 \\
\hline \multicolumn{2}{|l|}{ Discontinuation of treatment, $\mathrm{n}(\%)$} & $3(30.0 \%)$ & $1(6.6 \%)$ & 0.127 \\
\hline \multicolumn{2}{|l|}{ Adverse events } & $2(20.0 \%)^{a}$ & $1(6.6 \%)$ & \\
\hline \multicolumn{2}{|l|}{ Progression disease } & $1(10.0 \%)$ & $0(0.0 \%)$ & \\
\hline \multicolumn{2}{|l|}{ On day 180 after beginning therapy } & & Dose escalation $(n=14)$ & $P$ value \\
\hline Final dose: 100/200/300/400/500 mg QD, n & \multicolumn{2}{|c|}{ 0/0/5/1/1 (mean 343 mg/day) } & 1/2/4/4/3 (mean 346 mg/day) & 0.403 \\
\hline Median (range) duration of administration (days) & \multicolumn{2}{|c|}{$157.0(99-180)$} & $180.0(166-180)$ & $<0.001$ \\
\hline $\begin{array}{l}\text { Median (range) cumulative dose for } 6 \text { months } \\
\text { (mg) }\end{array}$ & \multicolumn{2}{|c|}{$51,700(32,800-90,000)$} & $53,550(29,700-75,600)$ & 1.000 \\
\hline $\begin{array}{l}\text { Median (range) dose intensity of bosutinib (mg/ } \\
\text { day) }\end{array}$ & \multicolumn{2}{|c|}{$309.8(182.2-500.0)$} & $295.0(165.0-420.0)$ & 1.000 \\
\hline $\begin{array}{l}\text { Median (range) plasma trough concentration } \\
(\mathrm{ng} / \mathrm{mL})\end{array}$ & \multicolumn{2}{|c|}{$63.7(31.9-126.0)($ mean $=75.1)$} & $63.0(31.4-113.0)($ mean $=65.8)$ & 0.588 \\
\hline
\end{tabular}

a Two patients discontinued bosutinib due to pancytopenia $(n=1)$ and elevation of lipase $(n=1)$ 
vortexed for $30 \mathrm{~s}$. This mixture was then applied to an Oasis HLB extraction cartridge that had been activated previously with methanol and water $(1.0 \mathrm{~mL}$ each). The cartridge was then washed with $1.0 \mathrm{~mL}$ of water and $1.0 \mathrm{~mL}$ of $60 \%$ methanol in water and eluted with $1.0 \mathrm{~mL}$ of $100 \%$ methanol. Eluates were dried by vortex-vacuum evaporation at $70{ }^{\circ} \mathrm{C}$ using a rotary evaporator (as-1 cve2as, Osaka, Japan). The resulting residue was then dissolved in $20 \mu \mathrm{L}$ of methanol and vortexed for $30 \mathrm{~s} ; 20 \mu \mathrm{L}$ of the mobile phase was added to the sample, and the sample was vortexed for another $30 \mathrm{~s}$. A $20 \mu \mathrm{L}$ aliquot of the sample was then processed by HPLC. The HPLC system comprised of a PU-2080 plus chromatography pump (Jasco, Tokyo, Japan) equipped with a capcell pak c18 mg $\Pi(250 \mathrm{~mm} \times 4.6 \mathrm{~mm}$ internal diameter, Shiseido, Tokyo, Japan) HPLC column, a UV-2075 light source, and an ultraviolet detector (Jasco). The mobile phase was $0.5 \%$ $\mathrm{KH}_{2} \mathrm{PO}_{4}(\mathrm{pH} 3.5)$-acetonitrile-methanol (60:35:5, v/v/v), which was degassed in an ultrasonic bath prior to use. The flow-rate was $0.5 \mathrm{~mL} / \mathrm{min}$ at ambient temperature and sample detection was conducted at $250 \mathrm{~nm}$.

The calibration line of bosutinib in plasma was linear over the concentration range of $10-500 \mathrm{ng} / \mathrm{mL}$. The extraction recovery value of bosutinib in this concentration range was $86-90 \%$. The coefficient of variations (CVs) and accuracies for the intra- and inter-day assays at concentrations of $10-500 \mathrm{ng} / \mathrm{mL}$ of bosutinib was less than $11.3 \%$ and within $9.8 \%$, respectively. The limit of quantification of bosutinib was $10 \mathrm{ng} / \mathrm{mL}$.

\section{Statistical analysis}

The Shapiro-Wilk test was used to assess the distribution of all patient data. The clinical characteristics of patients were expressed as the number or median value (range: minimum-maximum). The daily dose and $\mathrm{C}_{0}$ of bosutinib were expressed as the median and range. The Chi square test was used to examine the difference in categorical data. The Mann-Whitney U test was used to determine the difference in continuous variables between treatment groups. Spearman's rank correlation analysis was used to assess correlations with the $\mathrm{C}_{0}$, or the doseadjusted $\mathrm{C}_{0}$, of bosutinib with all results being expressed as correlation coefficients (r). Stepwise, multiple linear regression analysis was performed to determine the effect of all factors in the univariate analysis. The rate of side effects after beginning treatment between the two regimens was estimated using the Kaplan-Meier method and compared using the stratified log-rank test. $P$ values less than 0.05 were considered statistically significant. Statistical analyses were performed using SPSS version 20.0 for Windows (IBM Corp., Armonk, NY, USA).

\section{Results}

Clinical characteristics at the initial bosutinib administration (baseline) are shown in Table 1. There were no patients with serious renal or hepatic dysfunction. On the $\mathrm{SD}$ and $\mathrm{DE}$ regimens, the median patient age was 63.0 and 55.0 years, respectively, and the median body weight was 59.9 and $53.0 \mathrm{~kg}$, respectively. No significant difference was observed in any laboratory value at baseline between the two regimens, except for the hemoglobin value, which was associated with CML phase.

In nine of 10 patients $(90 \%)$ on the SD regimen, the bosutinib treatment was interrupted due to AEs. Subsequently, 8 patients were given a lower daily dose of bosutinib (Table 2). The median time to the first treatment interruption after beginning $500 \mathrm{mg} /$ day of bosutinib was 17.5 days. During 6 months, there was a median total interruption duration of 35 days. Moreover, after beginning the $500 \mathrm{mg} /$ day treatment, although dosage was adjusted based on the AE grade, 2 patients discontinued bosutinib therapy due to grade 3 elevation of lipase levels and grade 3 pancytopenia. In addition, another patient discontinued bosutinib therapy due to disease progression. Among 7 patients who were able to continue the bosutinib treatment, the final daily doses of bosutinib were $300 \mathrm{mg}$ (5 patients), $400 \mathrm{mg}$ (1 patient), and $500 \mathrm{mg}$ (1 patient), and the mean daily dose was of $343 \mathrm{mg} /$ day (median, $300 \mathrm{mg} /$ day). Only 1 patient could be treated during the 6-month period using the standard $500 \mathrm{mg}$ daily dose of bosutinib on a continuous basis. Consequently, the median $\mathrm{C}_{0}$ of bosutinib was $63.7 \mathrm{ng} / \mathrm{mL}$ (Table 2).

In 2 of 15 patients (13.3\%) on the DE regimen, the bosutinib treatment was interrupted due to liver dysfunction; however, after re-administration of bosutinib, 14 of 15 patients could be treated continuously during the treatment period after beginning the $100 \mathrm{mg} /$ day escalated dose. Only 1 patients showed symptoms due to the withdrawal of bosutinib, which included severe liver dysfunction (grade 4) for 14 days. The frequency of interruption was significantly lower in the DE than the SD regimen $(P<0.001)$. For the DE regimen, the final daily doses of bosutinib were $100 \mathrm{mg}$ (1 patient), $200 \mathrm{mg}(2$ patients), $300 \mathrm{mg}$ (4 patients), $400 \mathrm{mg}$ (4 patients), and $500 \mathrm{mg}$ (3 patients), with a mean dose of $346 \mathrm{mg} /$ day (median, $400 \mathrm{mg} /$ day) (Table 2). The cumulative dose of bosutinib for the entire treatment period was similar between the SD and DE treatment groups (51,700 mg vs. $53,550 \mathrm{mg}$, respectively). In addition, the median $\mathrm{C}_{0}$ was $63.0 \mathrm{ng} / \mathrm{mL}$, which was nearly identical to the $63.7 \mathrm{ng} / \mathrm{mL}$ value of the SD group.

In this study, 5 of 10 patients (50.0\%) experienced liver dysfunction (> grade 1) between days 19 and 57 (median, day 28 ) for the SD regimen, and 4 of 15 patients (26.7\%) 
Table 3 Appearance of liver dysfunction and diarrhea within $\mathbf{1 8 0}$ days after treatment

\begin{tabular}{|c|c|c|c|}
\hline Number of patients & Standard dose $(n=10)$ & Dose escalation $(n=15)$ & $P$ value \\
\hline Patient with liver dysfunction (> grade 1) (\%) & $5(50.0 \%)$ & $4(26.7 \%)$ & 0.234 \\
\hline Maximum grade (grade 2/3/4) & $4 / 1 / 0$ & $1 / 2 / 1$ & \\
\hline Day of the appearance of liver dysfunction (> grade 1), median (range) & $28(19-57)$ & $53.5(35-112)$ & \\
\hline $\begin{array}{l}\text { Cumulative dose until the appearance of liver dysfunction over grade } 2, \mathrm{mg} \text {, median } \\
\text { (range) }\end{array}$ & $8500(6500-20,500)$ & $11,300(5500-36,300)$ & 0.806 \\
\hline Dose at the appearance of liver dysfunction over grade 2, median (range) & $400(400-500)$ & $350(200-500)$ & 0.241 \\
\hline Discontinuation of bosutinib due to liver dysfunction (\%) & $0(0 \%)$ & $1(6.6 \%)$ & \\
\hline Patient with diarrhea (all grade) (\%) & $10(100 \%)$ & $11(73.3 \%)$ & 0.075 \\
\hline Maximum grade (grade $0 / 1 / 2 / 3 / 4$ ) & $0 / 4 / 3 / 3 / 0$ & $4 / 6 / 2 / 3 / 0$ & \\
\hline Day of the first appearance of diarrhea, median (range) & $1(1-5)$ & $19(1-148)$ & $<0.0001$ \\
\hline Dose at the first appearance of diarrhea (100/200/300/400/500 mg) & 0/0/0/0/10 & $4 / 3 / 1 / 3 / 0$ & \\
\hline Cumulative days of diarrhea, median (range) & $20.5(3-47)$ & $6(1-27)$ & 0.104 \\
\hline Discontinuation of bosutinib due to diarrhea (\%) & $0(0 \%)$ & $0(0 \%)$ & \\
\hline
\end{tabular}

experienced liver dysfunction between days 35 and 112 (median, day 53.5) in the DE regimen (Table 3). The Kaplan-Meier curve for the first appearance of liver dysfunction (>grade 1) is shown in Fig. 1a. Although the cumulative dose until the onset of liver dysfunction or the dose at the onset of liver dysfunction was not significant different between the SD and DE groups (Table 3), the cumulative incidence of liver dysfunction was significantly higher in the SD group $(P=0.029$, log-rank test). Differences in plasma $C_{0}$ between patients with liver dysfunction and patients without liver dysfunction were analyzed. The median plasma $C_{0}$ values were $96.1 \mathrm{ng} / \mathrm{mL}$ (range $37.2-148.0 \mathrm{ng} / \mathrm{mL}$ ) and $54.5 \mathrm{ng} / \mathrm{mL}$ (range 31.4$126.0 \mathrm{ng} / \mathrm{mL})$, respectively $(P=0.066$, Mann-Whitney $\mathrm{U}$ test) (Fig. 1b). Moreover, the bosutinib-induced liver dysfunction ( $\geq$ grade 1 , or $\geq$ grade 2 ) was also observed in patients with high bosutinib $C_{0}(>91.0 \mathrm{ng} / \mathrm{mL}$ of quartile 4 , calculated by the entire $\mathrm{C}_{0}$ distribution) (Table 4).

Similarly, after administration of bosutinib, 10 of 10 patients (100\%) experienced diarrhea between days 1 and 5 (median, day 1 ) on the SD regimen, as well as 11 of 15 (73.3\%) between days 1 and 148 (median, day 19) on the DE regimen (Table 3). The Kaplan-Meier curve for the appearance of bosutinib-induced diarrhea is shown in Fig. 1c. The cumulative incidence of diarrhea was significantly higher in the SD group $(P<0.001, \log$ rank test). No patient discontinued bosutinib therapy due to diarrhea in either regimen. However, 11 out of 25 patients presented with delayed/prolonged diarrhea, which occurred after day 14. Significant differences of plasma $C_{0}$ between patients with a delayed/ prolonged diarrhea (on the day when max grade diarrhea occurred) and patients without diarrhea (on day 180 ) were identified. The median plasma $C_{0}$ values were $103.5 \mathrm{ng} / \mathrm{mL}$ (range $58.3-144.5 \mathrm{ng} / \mathrm{mL}$ ) and $55.5 \mathrm{ng} /$
$\mathrm{mL}$ (range $31.4-126.0 \mathrm{ng} / \mathrm{mL}$ ), respectively $(P=0.007$, Mann-Whitney U test) (Fig. 1d). Moreover, diarrhea ( $\geq$ grade 2 , or $\geq$ grade 3 ) was observed in patients with high bosutinib $C_{0}(>91.0 \mathrm{ng} / \mathrm{mL}$ of quantile 4 , calculated by the entire $\mathrm{C}_{0}$ distribution) (Table 4 ). Although we tried to assess correlations between $\mathrm{C}_{0}$ of bosutinib and the clinical/laboratory data (age, sex, CML phase, reason of taking bosutinib, body weight, white blood cell count, hemoglobin levels, platelet count, aspartate transaminase, alanine transaminase, serum albumin levels, total bilirubin, serum creatinine levels, lipase value), there were no independent factors of the clinical characteristics influencing $\mathrm{C}_{0}$ (data not shown).

\section{Discussion}

In the present study, the DE regimen, where dose was increased by $100 \mathrm{mg}$ every 2 weeks, avoided treatment interruptions caused by adverse events, as compared to the SD regimen of the initial bosutinib dose of $500 \mathrm{mg} /$ day. There were significant differences in the frequency of treatment interruption $(90.0 \%$ vs. $13.3 \%, P<0.001)$, as well as the duration of interruption of bosutinib (35 days vs. 14 days, $P=0.013$ ), between the two regimens. In the SD regimen, $100 \%$ of patients had diarrhea within 5 days after beginning $500 \mathrm{mg} /$ day of bosutinib and $50 \%$ of patients had liver dysfunction (> grade 1 ) by day 57 after beginning this regimen. This phenomenon was consistent with previous reports [1-3, 9-12]. However, diarrhea just after the initiation of bosutinib was not observed in patients on the DE regimen, except one patient $(P<0.0001)$, with $26.7 \%$ of patients on the DE regimen showing liver dysfunction (> grade 1 ) by day 112 . There was a tendency of fewer patients showing liver dysfunction, or exhibiting a later symptomatic onset, on the $\mathrm{DE}$ regimen compared with the SD regimen, but this was 


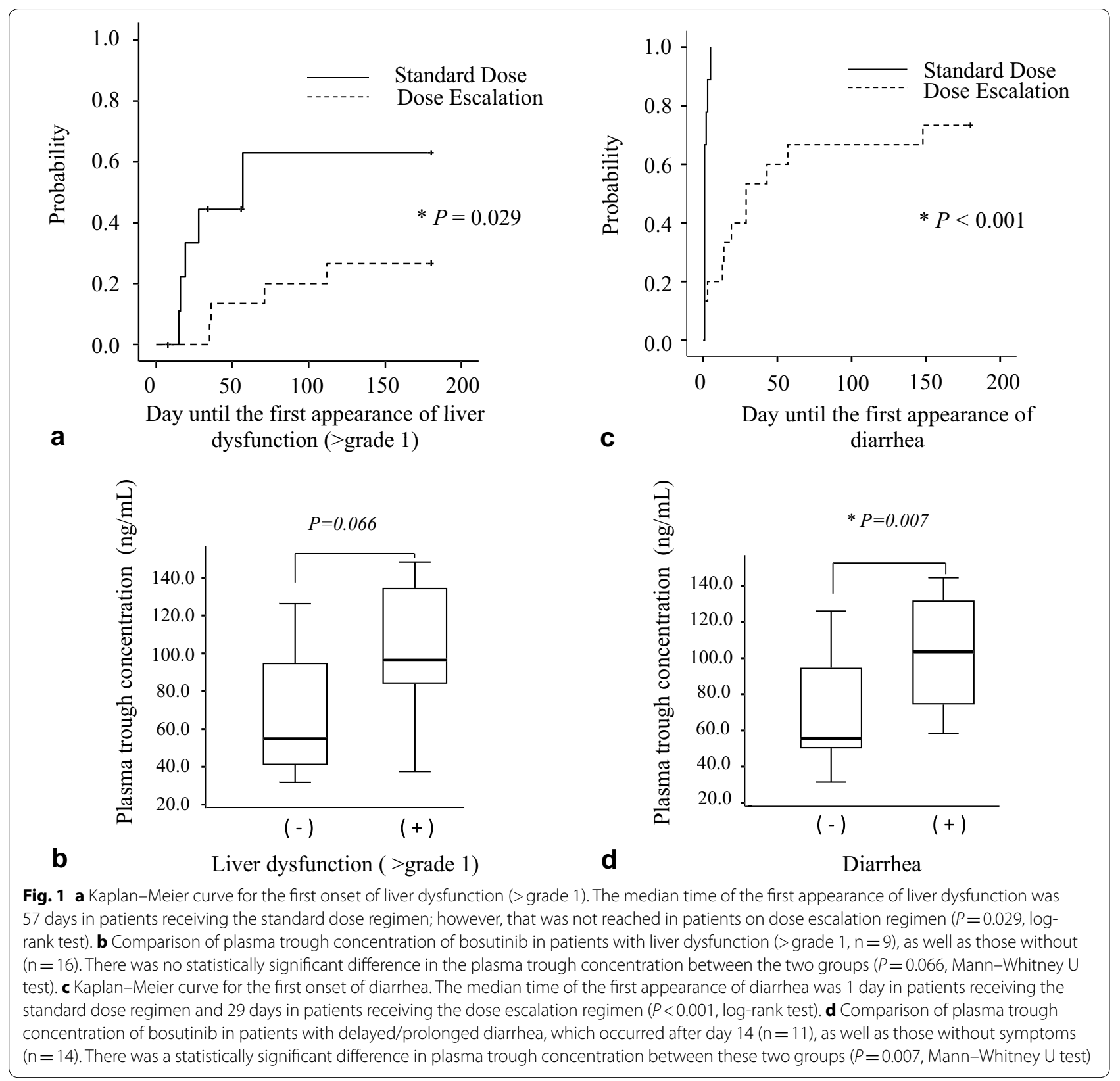

not statistically significant $(P=0.234)$. As a result, using bosutinib therapy with a DE regimen, we could administer continuous treatment without interruptions, due to a reduction in the onset or duration of AEs.

The final dose of bosutinib during the 6-month treatment duration for both regimens was 343 and $346 \mathrm{mg} /$ day, respectively, and the median cumulative dose during the treatment period was 51,700 and $53,550 \mathrm{mg}$, respectively. There were no significant dosimetric differences between the two treatment regimens. As a result of having adjusted the daily dose of bosutinib to minimize the incidence of adverse events, the median $\mathrm{C}_{0}$ during the treatment period for both regimens were the almost identical (63.7 and $63.0 \mathrm{ng} / \mathrm{mL}$, respectively). In a phase I/II study investigating Japanese CML-CP patients, the mean daily dose was $339.3 \mathrm{mg}$ (no data for $\mathrm{C}_{0}$ ) in a treatment period lasting 138 weeks [9], which is almost the same value as our present study.

This bosutinib $\mathrm{C}_{0}$ of $63 \mathrm{ng} / \mathrm{mL}$ in the present study was lower than the mean $\mathrm{C}_{0}$ of $156 \mathrm{ng} / \mathrm{mL}$ calculated by Hsyu et al. for an achievement of MMR at 12 months [8]. The mean $\mathrm{C}_{0}$ value reported by Hsyu et al. was obtained 
Table 4 Relationship between trough blood concentration and side effects observed in patients taking bosutinib

\begin{tabular}{lllll}
\hline & \multicolumn{3}{l}{ Distribution of $\mathbf{C}_{\mathbf{0}}$} & \\
\cline { 2 - 3 } & Q1 & Q2-3 & Q4 & \\
\hline Liver dysfunction & & & & \\
$\geq$ grade 1 & $6 / 34$ & $29 / 68$ & $15 / 34$ & 0.019 \\
$\geq$ grade 2 & $0 / 34$ & $2 / 68$ & $4 / 34$ & 0.050 \\
$\geq$ grade 3-4 & $0 / 34$ & $1 / 68$ & $2 / 34$ & 0.231 \\
Diarrhea & & & & \\
$\geq$ grade 1 & $5 / 34$ & $8 / 68$ & $6 / 34$ & 0.714 \\
$\geq$ grade 2 & $1 / 34$ & $2 / 68$ & $5 / 34$ & 0.041 \\
$\geq$ grade 3 & $1 / 34$ & $0 / 68$ & $4 / 34$ & 0.012 \\
\hline
\end{tabular}

Q quantile, $C_{0}$ trough blood concentration. Q1 ( $\left.\leq 32.5\right), \mathrm{Q} 2(>32.5), \mathrm{Q} 3(\leq 91.0)$, Q4 (>91.0). Median $\mathrm{C}_{0}=54.0(\mathrm{ng} / \mathrm{mL})$

from a phase III trial of newly diagnosed CML-CP within 3 months after the administration of $500 \mathrm{mg} /$ day of bosutinib [8]. Although the initial standard dose was $500 \mathrm{mg} /$ day in this trial, dose reductions of bosutinib as a result of AEs occurred in $39 \%$ of the study patients [4], and the median daily dose was $489 \mathrm{mg} /$ day $[4,8]$. The exposureresponse analysis for MMR and AEs have been reported for data from the BELA $(n=246)$ and BFORE $(n=266)$ trials [13]. The starting dose of bosutinib was $500 \mathrm{mg} /$ day (BELA) and $400 \mathrm{mg} /$ day (BFORE) [4, 5]. Although both trials had a higher proportion of Caucasian patients (79\% vs. 64\%) and a lower proportion of Asian patients (12\% vs. $26 \%$ ), the median $C_{0}$ values of bosutinib were $67.51 \mathrm{ng} / \mathrm{mL}\left(\mathrm{C}_{0}\right.$ range $\left.0.01-357.74\right)$ in the BELA trial and $55.75 \mathrm{ng} / \mathrm{mL}\left(\mathrm{C}_{0}\right.$ range $\left.0.01-223.83\right)$ in the BFORE trial [13], which was similar to our results. The MMR rate at 12 months was $38.0 \%$ in the BELA trial and $47.2 \%$ in the BFORE trial for de novo CML patients, which was significantly higher than the control treatment using imatinib $[4,5]$. Although we could not report any efficacy data in this study due to a short observation period, the importance of managing AEs in CML is underscored by the association between the $\mathrm{AE}$ occurrence and reduced treatment adherence. In addition, lower levels of adherence are associated with suboptimal treatment response [14]. The purpose of the dosage and treatment schedule investigated in our study was to reduce the occurrence of $\mathrm{AEs}$ and to avoid AE-related interruption in TKI treatment. A significantly higher number of patients were able to continue the bosutinib treatment in the DE regimen than the SD regimen. This result suggests that a higher level of adherence might be associated with an optimal treatment response.

The $\mathrm{C}_{0}$ value of $63 \mathrm{ng} / \mathrm{mL}$ may be appropriate in general clinical practice. The optimal therapeutic window for bosutinib is not yet determined; however, a target bosutinib $\mathrm{C}_{0}$ of $63 \mathrm{ng} / \mathrm{mL}$ in Japanese CML patients might be recommended to balance efficacy and toxicity. In addition, a bosutinib $C_{0}$ of more than $91.0 \mathrm{ng} /$ $\mathrm{mL}$ may be excessive for Japanese CML patients; the bosutinib-induced diarrhea $>$ grade 2 and liver dysfunction $>$ grade 1 were observed in patients with high bosutinib $\mathrm{C}_{0}(>91.0 \mathrm{ng} / \mathrm{mL})$.

Nakaseco et al. reported that bosutinib exposure on day 15 after administration is not different for daily doses of 500 and $600 \mathrm{mg}$ [9], and Cortes et al. also reported that there is no increase in bosutinib exposure on day 15 when the bosutinib dose increased from 400 to 500 $600 \mathrm{mg} /$ day (mean $\mathrm{AUC}_{0-24}: 2851 ; 3660$; and $3360 \mathrm{ng}$ "h/ $\mathrm{mL}$, respectively) [1]. These results show that the clinical response of bosutinib might depend on plasma concentration, but not the daily dose. In these reports, they explain that the solubility of bosutinib is saturated at a higher daily dose $[1,9]$. In the previous study, the interindividual variability of bosutinib was very large $(\% \mathrm{CV}$ of about 70\%) [8]. A possible causative factor for the large variability is a solubility of bosutinib due to a dependence on the intra-gastric $\mathrm{pH}$, and it could continuously change the absorption of the drug from the gastrointestinal tract [15]. TDM for bosutinib might be a useful strategy for dose optimization to obtain a faster and deeper clinical response, due to the wide distribution of plasma concentrations for bosutinib.

The efficacy and safety of a dose-escalation schedule was confirmed by the bosutinib concentration level used in this study. The cost of the HPLC method for measuring the concentration of bosutinib is reasonable ( $\$ 4$ per sample, including running costs of HPLC) and it is easy to perform at any institute as demonstrated in the methods section [16]. However, the dosage of bosutinib can increase, according to the DE regimen, without measuring the concentration of bosutinib in daily practice. If patients show any AEs, even for low-grade AEs in the DE regimen, the dosage of bosutinib should be at the same level and treatment can be continued without interruption. If a clinician can monitor bosutinib $\mathrm{C}_{0}$, as provisionally defined by the ELN, simultaneously with an evaluation of clinical response according to transcript levels at each time point, then the mean bosutinib $C_{0}$ should also be assessed for 3 months, or using multiple points, for each patient treated with bosutinib. In addition, after changing bosutinib dose, it may be necessary to monitor bosutinib $\mathrm{C}_{0}$ for approximately 1 week (day 8).

The limitation of this study was the small sample size. Further examination with a larger sample size will be necessary to evaluate the relationships between the pharmacokinetics of bosutinib and various AEs. However, individualization of the daily dose of bosutinib, based on $\mathrm{C}_{0}$, 
might be useful, as well as the TDM for other TKIs such as imatinib, nilotinib, dasatinib, and ponatinib [6, 17].

\section{Conclusions}

The DE regimen was better than the SD regimen at avoiding treatment interruptions due to AEs. The daily dose of bosutinib might be adjusted based on the target $\mathrm{C}_{0}$ to avoid adverse events by TDM in general clinical practice.

\begin{abstract}
Abbreviations
TKI: tyrosine kinase inhibitor; Abl: Abelson; CP: chronic phase; CML: chronic myeloid leukemia; BELA: Bosutinib Efficacy and Safety in Newly Diagnosed CML; BFORE: First-line chrOnic myelogenous leukemia tREatment; MMR: major molecular response; FDA: Food and Drug Administration; TDM: therapeutic drug monitoring; AEs: adverse events; $\mathrm{C}_{0}$ : trough plasma concentration; SD: standard dose; DE: dose escalation; CTCAE: Common Terminology Criteria for Adverse Events; HPLC: high-performance liquid chromatography; CVs: coefficient of variations.
\end{abstract}

\section{Authors' contributions}

$\mathrm{AM}, \mathrm{MM}$, and NT participated in the design of the study and drafted the manuscript. AM, MA, ST, and TY collected clinical information. MA, TN and MM carried out the pharmacokinetic studies. AM, MA, TN, MM, and NT performed the statistical analysis. MA, TN, ST, and YK participated in its design and coordination, and helped to draft the manuscript. All authors read and approved the final manuscript.

\section{Author details \\ ${ }^{1}$ Department of Hematology, Nephrology, and Rheumatology, Akita Univer- sity Graduate School of Medicine, 1-1-1 Hondo, Akita City, Akita 010-8543, Japan. ${ }^{2}$ Department of Pharmacy, Akita University Hospital, Akita, Japan. ${ }^{3}$ Clinical Research Promotion and Support Center, Akita University Hospital, Akita, Japan}

\section{Acknowledgements}

Not applicable.

\section{Competing interests}

NT received research funding and honoraria for lectures from Pfizer and Novartis. The other authors declare that they have no competing interests.

\section{Availability of data and materials}

The datasets analyzed during the current study are available from the corresponding author on reasonable request.

\section{Consent for publication}

Not applicable. This manuscript does not contain any individual person's data in any form (including individual details, images or videos). Consent to publish has been obtained from the participant.

\section{Ethics approval and consent to participate}

This study was approved by the Ethics Committee of Akita University Graduate School of Medicine (No. 1140). Each participant provided informed consent and signed a human subject institutional review board consent form.

\section{Funding}

This work was supported by a grant (Nos. 26927001, 26460188, 17K08408) from the Japan Society for the Promotion of Science, Tokyo, Japan.

\section{Publisher's Note}

Springer Nature remains neutral with regard to jurisdictional claims in published maps and institutional affiliations.

Received: 31 January 2018 Accepted: 10 April 2018

Published online: 13 April 2018
References

1. Cortes JE, Kantarjian HM, Brummendorf TH, Kim DW, Turkina AG, Shen ZX, et al. Safety and efficacy of bosutinib (SKI-606) in chronic phase Philadelphia chromosome-positive chronic myeloid leukemia patients with resistance or intolerance to imatinib. Blood. 2011;118:4567-76.

2. Khoury HJ, Cortes JE, Kantarjian HM, Gambacorti-Passerini C, Baccarani $\mathrm{M}$, Kim DW, et al. Bosutinib is active in chronic phase chronic myeloid leukemia after imatinib and dasatinib and/or nilotinib therapy failure. Blood. 2012;119:3403-12.

3. Kantarjian HM, Cortes JE, Kim DW, Khoury HJ, Brümmendorf TH, Porkka K, et al. Bosutinib safety and management of toxicity in leukemia patients with resistance or intolerance to imatinib and other tyrosine kinase inhibitors. Blood. 2014;123:1309-18.

4. Cortes JE, Kim DW, Kantarjian HM, Brümmendorf TH, Dyagil I, Griskevicius $L$, et al. Bosutinib versus imatinib in newly diagnosed chronic-phase chronic myeloid leukemia: results from the BELA trial. J Clin Oncol. 2012;30:3486-92.

5. Cortes JE, Gambacorti-Passerini C, Deininger MW, Mauro MJ, Chuah C, Kim DW, et al. Bosutinib versus imatinib for newly diagnosed chronic myeloid leukemia: results from the randomized BFORE trial. J Clin Oncol. 2018;36:231-7.

6. Miura M. Therapeutic drug monitoring of imatinib, nilotinib, and dasatinib for patients with chronic myeloid leukemia. Biol Pharm Bull. 2015;38:645-54.

7. Hsyu PH, Mould DR, Upton RN, Amantea M. Pharmacokinetic-pharmacodynamic relationship of bosutinib in patients with chronic phase chronic myeloid leukemia. Cancer Chemother Pharmacol. 2013;71:209-18.

8. Hsyu PH, Mould DR, Abbas R, Amantea M. Population pharmacokinetic and pharmacodynamic analysis of bosutinib. Drug Metab Pharmacokinet. 2014;29:441-8.

9. Nakaseko C, Takahashi N, Ishizawa K, Kobayashi Y, Ohashi K, Nakagawa Y, et al. A phase 1/2 study of bosutinib in Japanese adults with Philadelphia chromosome-positive chronic myeloid leukemia. Int J Hematol. 2015;101:154-64.

10. Takahashi N, Nakaseko C, Kobayashi Y, Miyamura K, Ono C, Koide Y, et al. Long-term treatment with bosutinib in a phase $1 / 2$ study in Japanese chronic myeloid leukemia patients resistant/intolerant to prior tyrosine kinase inhibitor treatment. Int J Hematol. 2017;106:398-410.

11. Gambacorti-Passerini C, Brummendorf TH, Kim DW, Turkina AG, Masszi T, Assouline S, et al. Bosutinib efficacy and safety in chronic phase chronic myeloid leukemia after imatinib resistance or intolerance: minimum 24-month follow-up. Am J Hematol. 2014;89:732-42.

12. Gambacorti-Passerini C, Cortes JE, Lipton JH, Dmoszynska A, Wong RS, Rossiev V, et al. Safety of bosutinib versus imatinib in the phase 3 BELA trial in newly diagnosed chronic phase chronic myeloid leukemia. Am J Hematol. 2014;89:947-53.

13. Garrett M, Knight B, Cortes JE, Deininger MW. Optimizing dose of bosutinib to minimize adverse events while maintaining efficacy in patients with newly diagnosed chronic myelogenous leukemia. Blood (ASH Annual Meeting Abstracts). 2017;130:2899.

14. Marin D, Bazeos A, Mahon FX, Eliasson L, Milojkovic D, Bua M, et al. Adherence is the critical factor for achieving molecular responses in patients with chronic myeloid leukemia who achieve complete cytogenetic responses on imatinib. J Clin Oncol. 2010;28:2381-8.

15. Abbas R, Hug BA, Leister C, Gaaloul ME, Chalon S, Sonnichsen D. A phase I ascending single-dose study of the safety, tolerability, and pharmacokinetics of bosutinib (SKI-606) in healthy adult subjects. Cancer Chemother Pharmacol. 2012;69:221-7.

16. Miura M, Takahashi M. Routine therapeutic drug monitoring of tyrosine kinase inhibitors by HPLC-UV or LC-MS/MS methods. Drug Metab Pharmacokinet. 2016;31:12-20.

17. Abumiya M, Miura M, Takahashi N. Therapeutic drug monitoring of ponatinib using a simple high-performance liquid chromatography method in Japanese patients. Leuk Res. 2017;64:42-5. 